\title{
Perfil do idoso dependente de cuidado domiciliar em comunidades de baixo nível socioeconômico de Porto Alegre, Rio Grande do Sul
}

\author{
Profile of the elderly individual dependent on home care \\ in low socioeconomic level communities in Porto Alegre \\ in the State of Rio Grande do Sul
}

Giovâni Firpo Del Duca ${ }^{1}$

Aline de Deus Martinez ${ }^{2}$

Gisele Alsina Nader Bastos ${ }^{3}$

${ }^{1}$ Programa de Pósgraduação em Educação Física, Universidade Federal de Santa Catarina. Campus

Universitário, Coordenadoria de PósGraduação em Educação Física, Bairro Trindade. 88040-900 Florianópolis Santa Catarina. gfdelduca@gmail.com

${ }^{2}$ Programa de Pós Graduação em Medicina de Família e Comunidade, Escola de Gestão em Saúde, Hospital Moinhos de Vento. ${ }^{3}$ Escola de Gestão em Saúde, Hospital Moinhos de Vento.
Abstract The scope of this study was to estimate the prevalence of home care and to identify the demographic, socioeconomic, behavioral and health profile of dependent elderly individuals. The cross-sectional and representative delineation of the communities of Restinga and Extremo Sul in Porto Alegre in the State of Rio Grande do Sul included 638 elderly individuals. Home care was defined as a positive answer to the following question: "Do you have someone here in your home to take care of you?" The prevalence of home care was $49.5 \%$ (95\% CI: 44.5; 54.5). Children and spouses were responsible for the majority of home care $(40.7 \%$ and $37 \%$, respectively). Men, unaccompanied and physically inactive individuals, of advanced age, lower educational level, higher economic level, functionally disabled and who had been hospitalized in the last year characterized the profile of the dependent elderly individual. The high prevalence of home care can serve as input for health services for the elderly. Among all the characteristics, functional disability was the one most closely associated with home care.

Key words Elderly, Caregivers, Home nursing, Socioeconomic factors, Aging
Resumo O estudo estimou a prevalência de cuidado domiciliar e identificou o perfil demográfico, socioeconômico, comportamental e de saúde dos idosos dependentes. O delineamento transversal e representativo das comunidades Restinga e Extremo Sul, de Porto Alegre (RS), incluiu 638 idosos. O cuidado domiciliar foi definido pela resposta positiva à questão: "O(a) Sr. (a) tem alguém aqui na sua casa para cuidar do(a) Sr(a)?”. A prevalencia de cuidado domiciliar encontrada foi de 25,4\% (IC95\%: 22,0; 28,8). Filhos(as) e esposos(as) foram responsáveis pela maioria do cuidado domiciliar prestado a idosos $(40,7 \%$ e $37,0 \%$, respectivamente). Homens, individuos sem companheiro(a), fisicamente inativos, com idade avançada, menor escolaridade e maior nível econômico, incapacidade funcional e que foram hospitalizados no último ano caracterizaram o perfil do idoso dependente. A alta prevalência de cuidado domiciliar serve de subsídio para politicas públicas destinadas a idosos. Dentre todas as características avaliadas, a incapacidade funcional, esteve mais fortemente associada ao cuidado domiciliar.

Palavras-chave Idoso, Cuidadores, Assistência domiciliar, Fatores socioeconômicos, Envelhecimento 


\section{Introdução}

O aumento da expectativa de vida e a diminuição das taxas de fecundidade são aspectos marcantes da chamada transição demográfica. De modo geral, observa-se um aumento da população de idosos de forma mais acentuada nos países em desenvolvimento, embora este contingente ainda seja proporcionalmente bem inferior ao encontrado nos desenvolvidos ${ }^{1}$. Especificamente no Brasil, estima-se que em 2025 os idosos atingirão o número de 33,4 milhões, passando a ocupar a quinta posição entre os países com maior número absoluto de indivíduos com 60 anos de idade ou mais².

O processo de envelhecimento é marcado por profundas mudanças no perfil de saúde. Em idosos, a ocorrência das chamadas doenças crônicas, quedas e da incapacidade funcional tendem a aumentar com o avanço da idade, impedindo em muitos casos a total independência e autonomia do indivíduo ${ }^{3-5}$, fazendo com que necessitem, em alguns casos, de cuidados periódicos em seu domicílio com a supervisão constante de um familiar, ou mesmo de um profissional capacitado.

Os cuidadores de idosos são geralmente responsáveis por auxiliá-los em atividades do cotidiano, como alimentação, higiene pessoal, medicação de rotina e deslocamentos, colaborando para a recuperação, ou mesmo na estabilização da qualidade de vida ${ }^{6}$. Em regiões em desenvolvimento, os cuidadores informais assumem grande responsabilidade pelo cuidado ao idoso. No Brasil, a Política Nacional da Saúde do Idoso e o Estatuto do Idoso ${ }^{8}$ preconizam a permanência dos idosos sob os cuidados de suas famílias pelo maior tempo possível, desestimulando ações como a institucionalização. No entanto, devido à sobrecarga física e ao desgaste emocional, o cuidador informal pode se tornar mais vulnerável, especialmente quando passa a responsabilizar-se por um familiar próximo em situação de fragilidade 9.

Diante da problemática apresentada, este trabalho teve por objetivo estimar a prevalência de cuidado domiciliar e identificar o perfil demográfico, socioeconômico, comportamental e de saúde de idosos dependentes em comunidades de baixo nível socioeconômico de Porto Alegre, Rio Grande do Sul.

\section{Métodos}

Este estudo está vinculado a amplo inquérito epidemiológico desenvolvido nos distritos sanitári- os da Restinga e Extremo Sul, em Porto Alegre, Rio Grande do Sul. Os bairros Lajeado, Lami, Belém Novo, Ponta Grossa e Chapéu do Sol formam o chamado Extremo Sul. Já a Restinga é um dos maiores bairros da capital gaúcha e ambas as localidades possuem um grande contingente populacional com baixo nível socioeconômico.

O delineamento adotado foi transversal de base populacional. No processo amostral por conglomerados, foram definidos dois estágios: os setores censitários, definidos como unidades amostrais primárias e os domicílios, constituindo as unidades amostrais secundárias. Foi realizado mapeamento prévio dos 121 setores censitários existentes, constatando-se que, destes, 117 eram setores domiciliares. $\mathrm{Na}$ fase de identificação de domicílios, foram mapeadas 29.929 residências habitadas. A partir de pulos sistemáticos, fizeram parte da amostra todos os indivíduos com idade igual ou superior a 60 anos residentes nos domicílios sorteados. Perdas e recusas foram definidas após a não realização da entrevista em, no mínimo, três visitas efetuadas em dias e horários distintos.

O cálculo de tamanho amostral utilizou os seguintes parâmetros e estimativas: prevalência de cuidado domiciliar de 25\%, intervalo de confiança de 95\% (IC95\%) e erro aceitável de quatro pontos percentuais, com adicional de $10 \%$ para perdas e recusas, resultando em 495 sujeitos. No entanto, outros cálculos de amostra também foram realizados e, em função dos diversos desfechos estudados no inquérito, prevalecendo o maior tamanho de amostra.

A coleta de dados foi realizada no período de julho a dezembro de 2009. As entrevistas foram conduzidas por entrevistadores submetidos a treinamento de 80 horas para a devida aplicação do questionário. Nos casos em que o idoso apresentasse incapacidade para responder o questionário, as informações eram obtidas por proxy, a partir do relato do(a) cuidador(a). Foi realizado o controle de qualidade em $10 \%$ das entrevistas via telefone. Para digitalização dos dados e checagem das inconsistências utilizou-se o programa Office Remark (Gravic Inc, Filadélfia, Estados Unidos).

O desfecho do estudo foi o cuidado domiciliar, avaliado a partir da seguinte questão: " $\mathrm{O}(\mathrm{a})$ Sr.(a) tem alguém aqui na sua casa para cuidar do(a) Sr(a)?". Já as exposições do estudo foram categorizadas do seguinte modo: sexo (masculino e feminino); idade em anos completos (6069, 70-79 e $\geq 80$ ); situação conjugal atual - com companheiro(a), solteiro(a) e separado(a)/viú- 
vo(a); escolaridade em anos completos de estudo (0-4, 5-11 e $\geq 12$ ); nível econômico em quartis, a partir da aplicação do questionário padronizado da Associação Brasileira de Empresas de Pesquisa ${ }^{10}$; nível de atividade física (inativo, pouco ativo ou ativo, correspondendo às categorias de 0 minuto/semana, 10 a 149 minutos/semana e $\geq 150$ minutos/semana de prática de atividade física conforme o questionário Internacional de Atividade Física) ${ }^{11}$; incapacidade funcional para atividades básicas da vida diária, empregandose o Índice de Katz ${ }^{12}$ e definindo-se como a necessidade de ajuda parcial ou total para, no mínimo, uma das atividades diárias investigadas (tomar banho, vestir-se, ir ao banheiro, deitar e levantar da cama/cadeira, comer e controle das funções de urinar/evacuar); hospitalização no último ano (sim ou não) e autopercepção de saúde (muito boa/boa, regular e ruim/muito ruim).

$\mathrm{Na}$ estatística descritiva, foram empregadas proporções e respectivos IC95\% para variáveis categóricas, assim como médias, amplitudes de variação e desvios-padrão (dp) para variáveis numéricas. Na análise bruta, empregou-se o teste qui-quadrado de Pearson para variáveis categóricas dicotômicas e politômicas nominais e tendência linear para variáveis categóricas ordinais. $\mathrm{Na}$ análise ajustada, foi empregada a regressão de Poisson com variância robusta, com os resultados expressos em razões de prevalências ${ }^{13}$. Levou-se em consideração a hierarquia entre os possíveis fatores associados com o desfecho para a análise dos dados: sexo, idade e situação conjugal foram considerados determinantes distais; escolaridade e nível econômico inseridos no nível dois; nível de atividade física foi incluído no nível três; hospitalização no último ano e incapacidade funcional foram agrupados em um quarto nível, e como determinante proximal a autopercepção de saúde. Na modelagem estatística, adotou-se a estratégia de seleção "para trás” e um nível crítico de valor $\mathrm{p} \leq 0,20$ para permanência no modelo para controle de confusão, considerando-se estatisticamente significativos valores $\mathrm{p} \leq 0,05$.

O protocolo de pesquisa foi aprovado pelo Comitê de Ética em Pesquisa do Hospital Moinhos de Vento.

\section{Resultados}

Nos domicílios visitados, 671 sujeitos foram elegíveis para o estudo (idade $\geq 60$ anos). Destes, foram entrevistados 638 idosos, o que corres- pondeu a um percentual de perdas/recusas de 4,9\%. Do total de questionários aplicados no estudo, 7,8\% foram respondidos com auxílio do cuidador, em função da incapacidade de o idoso relatar respostas válidas.

A idade da amostra variou de 60 a 96 anos (média $=68,6$ e dp $=7,1$ ), sendo a maioria dos entrevistados do sexo feminino $(59,7 \%)$. Considerável proporção de idosos tinha a situação conjugal de separado ou viúvo $(39,2 \%)$ e possuía escolaridade de 0 a 4 anos de estudo (48,9\%). Em relação aos aspectos de saúde, 66,3\% eram inativos, $15,7 \%$ apresentavam incapacidade funcional e $16,1 \%$ foram hospitalizados no último ano (Tabela 1 ).

Tabela 1. Análise descritiva dos idosos moradores dos bairros Restinga e Extremo Sul. Porto Alegre, Rio Grande do Sul, 2009.

\begin{tabular}{|c|c|c|}
\hline Variável & $\mathbf{n}$ & $\%$ \\
\hline \multicolumn{3}{|l|}{ Sexo } \\
\hline Masculino & 257 & 40,3 \\
\hline Feminino & 381 & 59,7 \\
\hline \multicolumn{3}{|l|}{ Idade (anos completos) } \\
\hline $60-69$ & 403 & 63,2 \\
\hline $70-79$ & 180 & 28,2 \\
\hline$\geq 80$ & 55 & 8,6 \\
\hline \multicolumn{3}{|l|}{ Situação conjugal atual } \\
\hline Com companheiro(a) & 334 & 52,3 \\
\hline Solteiro(a) & 54 & 8,5 \\
\hline Separado(a)/Viúvo(a) & 250 & 39,2 \\
\hline \multicolumn{3}{|c|}{ Escolaridade (anos completos) } \\
\hline $0-4$ & 312 & 48,9 \\
\hline $5-11$ & 222 & 34,8 \\
\hline$\geq 12$ & 104 & 16,3 \\
\hline \multicolumn{3}{|c|}{ Nível econômico (em quartis) ${ }^{\mathrm{a}}$} \\
\hline $1^{\circ}$ quartil (mais pobre) & 181 & 28,7 \\
\hline $2^{\circ}$ quartil & 147 & 23,3 \\
\hline $3^{\circ}$ quartill & 157 & 24,9 \\
\hline $4^{\circ}$ quartil (mais rico) & 146 & 23,1 \\
\hline \multicolumn{3}{|c|}{ Nível de atividade física (min/sem) } \\
\hline Inativos $(\leq 149)$ & 423 & 66,3 \\
\hline Ativos $(\geq 150)$ & 215 & 33,7 \\
\hline \multicolumn{3}{|l|}{ Incapacidade funcional } \\
\hline Não & 536 & 84,3 \\
\hline Sim & 100 & 15,7 \\
\hline \multicolumn{3}{|l|}{ Hospitalização } \\
\hline Não & 535 & 83,9 \\
\hline Sim & 103 & 16,1 \\
\hline \multicolumn{3}{|l|}{ Autopercepção de saúde } \\
\hline Muito boa/boa & 266 & 41,8 \\
\hline Regular & 283 & 44,5 \\
\hline Ruim/muito ruim & 87 & 13,7 \\
\hline
\end{tabular}

a Variável com maior número de valores ignorados $(\mathrm{n}=7)$. 
A prevalência de cuidado domiciliar encontrada foi de 25,4\% (IC95\%: 22,0;28,8). A maioria dos cuidadores eram entes da própria família: filho(a) $-40,7 \%$ e esposo(a) $-37,0 \%$ foram as respostas mais relatadas pelos entrevistados. Apenas $4,3 \%$ dos idosos disseram ter cuidador formal contratado.

A Tabela 2 apresenta os fatores associados ao cuidado domiciliar em idosos moradores da Res-
tinga/Extremo Sul. Após análise ajustada, constatou-se que homens tiveram uma probabilidade $50 \%$ maior de terem cuidador no domicílio. Os idosos sem companhia, tanto aqueles solteiros $(\mathrm{RP}=1,61)$, quanto os separados e os viúvos $(\mathrm{RP}=1,45)$ apresentaram maiores prevalências de cuidado domiciliar. O desfecho esteve também associado ao avanço da idade $(\mathrm{p}<0,001)$, ao menor nível de escolaridade $(\mathrm{p}=0,003)$ e ao

Tabela 2. Prevalência, análise bruta e ajustada do cuidado domiciliar em idosos moradores da Restinga/Extremo Sul conforme variáveis independentes. Porto Alegre, Rio Grande do Sul, 2009.

\begin{tabular}{|c|c|c|c|c|c|}
\hline \multirow{2}{*}{ Variáveis } & \multirow{2}{*}{$\begin{array}{c}\text { Cuidado } \\
\text { domiciliar (\%) }\end{array}$} & \multicolumn{2}{|c|}{ Análise Bruta } & \multicolumn{2}{|c|}{ Análise Ajustada } \\
\hline & & $\mathbf{R P}\left(\mathrm{IC}_{95 \%}\right)$ & $\mathrm{p}$ & $\operatorname{RP}\left(\mathrm{IC}_{95 \%}\right)$ & $\mathrm{p}$ \\
\hline \multicolumn{6}{|l|}{ Bloco 1: Variáveis demográficas } \\
\hline Sexo & & & 0,29 & & 0,006 \\
\hline Masculino & 27,6 & $1,16(0,89 ; 1,51)$ & & $1,50(1,12 ; 2,00)$ & \\
\hline Feminino & 23,9 & 1,00 & & 1,00 & \\
\hline Idade (anos completos) & & & $<0,001^{\star}$ & & $<0,001^{\star}$ \\
\hline $60-69$ & 16,6 & 1,00 & & 1,00 & \\
\hline $70-79$ & 35,6 & $2,14(1,59 ; 2,87)$ & & $2,01(1,49 ; 2,71)$ & \\
\hline$\geq 80$ & 56,4 & $3,39(2,46 ; 4,67)$ & & $3,21(2,30 ; 4,48)$ & \\
\hline Situação conjugal & & & 0,01 & & 0,04 \\
\hline Com companheiro(a) & 20,4 & 1,00 & & 1,00 & \\
\hline Solteiro(a) & 31,5 & $1,55(0,99 ; 2,41)$ & & $1,61(1,01 ; 2,55)$ & \\
\hline Separado(a)/Viúvo(a) & 30,8 & $1,51(1,14 ; 2,00)$ & & $1,45(1,06 ; 1,97)$ & \\
\hline \multicolumn{6}{|l|}{ Bloco 2: Variáveis socioeconômicas } \\
\hline Escolaridade (anos completos) & & & $0,001^{*}$ & & $0,003^{*}$ \\
\hline $0-4$ & 56,4 & 1,00 & & 1,00 & \\
\hline $5-11$ & 35,6 & $0,60(0,44 ; 0,83)$ & & $0,69(0,50 ; 0,94)$ & \\
\hline$\geq 12$ & 16,6 & $0,57(0,37,0,88)$ & & $0,56(0,36,0,88)$ & \\
\hline Nível econômico (ABEP) & & & $0,09^{\star}$ & & $0,001^{\star}$ \\
\hline $1^{\circ}$ quartil (mais pobre) & 21,6 & 1,00 & & 1,00 & \\
\hline $2^{\circ}$ quartil & 23,1 & $1,07(0,72 ; 1,61)$ & & $1,28(0,86 ; 1,92)$ & \\
\hline $3^{\circ}$ quartil & 30,6 & $1,42(0,99 ; 2,04)$ & & $1,61(1,14 ; 2,29)$ & \\
\hline $4^{\circ}$ quartil (mais rico) & 27,4 & $1,27(0,87 ; 1,87)$ & & $1,78(1,22 ; 2,61)$ & \\
\hline \multicolumn{6}{|l|}{ Bloco 3: Variáveis comportamentais } \\
\hline Nível de atividade física (min/sem) & & & 0,007 & & 0,05 \\
\hline Inativos $(\leq 149 \mathrm{~min} / \mathrm{sem})$ & 28,8 & 1,00 & & 1,00 & \\
\hline Ativos $(\geq 150 \mathrm{~min} / \mathrm{sem})$ & 18,6 & $0,65(0,47 ; 0,89)$ & & $0,73(0,52 ; 1,00)$ & \\
\hline \multicolumn{6}{|l|}{ Bloco 4: Variáveis de saúde } \\
\hline Incapacidade funcional & & & $<0,001$ & & $<0,001$ \\
\hline Não & 18,1 & 1,00 & & 1,00 & \\
\hline $\operatorname{Sim}$ & 64,0 & $3,54(2,80 ; 4,46)$ & & $2,70(2,09 ; 3,50)$ & \\
\hline Hospitalização & & & $<0,001$ & & 0,02 \\
\hline Não & 22,2 & 1,00 & & 1,00 & \\
\hline $\operatorname{Sim}$ & 41,8 & $1,88(1,42 ; 2,48)$ & & $1,38(1,06 ; 1,79)$ & \\
\hline \multicolumn{6}{|l|}{ Bloco 5: Variável de autopercepção } \\
\hline Autopercepção de saúde & & & $<0,001^{\star}$ & & 0,28 \\
\hline Muito boa/boa & 21,1 & 1,00 & & 1,00 & \\
\hline Regular & 22,3 & $1,06(0,77 ; 1,45)$ & & $0,94(0,69 ; 1,27)$ & \\
\hline Ruim/muito ruim & 47,1 & $2,24(1,62 ; 3,09)$ & & $1,27(0,89 ; 1,79)$ & \\
\hline
\end{tabular}

* Tendência linear 
maior nível econômico ( $\mathrm{p}=0,001)$. Idosos ativos tiveram uma probabilidade $27 \%$ menor de cuidado domiciliar, enquanto aqueles com incapacidade funcional e que foram hospitalizados no último ano apresentaram riscos de 2,70 e 1,38, respectivamente, para a ocorrência do desfecho.

\section{Discussão}

O incremento da expectativa de vida consiste em um fenômeno global responsável por gerar diversas repercussões à assistência aos idosos, desde o cuidado direto intradomiciliar até a necessidade de maiores investimentos nas políticas de saúde pública, tais como ampliação da rede de atenção básica voltada a eles. A frequente necessidade de cuidados médicos, o uso de medicamentos, as internações e os agravos de saúde decorrentes de incapacidade funcional ${ }^{14}$ repercutem na saúde pública, onde percebe-se que os investimentos na área de saúde do idoso, dependente ou não de cuidado domiciliar, parecem insuficientes frente à crescente demanda de necessidades em saúde atribuídas a esta faixa etária ${ }^{15}$.

A prevalência de idosos com cuidados no domicílio encontrada neste estudo foi de 25,4\% (IC95\%: 22,0; 28,8). Pesquisas nesta temática encontram prevalências entre $23,0 \%{ }^{16}$ e $49,5 \%{ }^{17}$. Esta variação percentual justifica-se na adoção de diferentes critérios para determinar o cuidado domiciliar, visto que não há instrumento padrão para mensurá-lo, bem como nas diferentes características socioeconômicas das populações avaliadas, o que limita maiores comparações entre estudos.

O presente estudo ratificou o predomínio de cuidados prestados por filhos(as) e cônjuges aos idosos em seus domicílios. Existe uma alternância entre várias pesquisas no estabelecimento de vínculo familiar com o cuidador: em alguns, há maioria de cuidadores na posição de filhos ${ }^{18}$; em outros, a situação de cônjuge é mais frequente ${ }^{16,17}$. Embora a dinâmica familiar seja transformada pela presença de um idoso dependente, nota-se que esta permanece como a opção mais viável de prestação de cuidados, seja pelo fator financeiro ou pela força cultural da obrigação familiar. Frente à possibilidade de transferir os cuidados para instituições de longa permanência, tanto familiares ${ }^{19}$, quanto idosos ${ }^{20}$ e políticas públicas em vigor ${ }^{8}$ afirmam que esta seria uma alternativa para casos críticos. Porém, é preciso atentar que a falta de informações necessárias para lidar com doenças crônico-degenerativas, e a ausência de suporte para lidar com as mudanças emocionais geradas pelo surgimento de incapacidades, tornam os cuidadores mais vulneráveis ao adoecimento ${ }^{6,9}$.

Diferentemente de outros trabalhos ${ }^{16,21}$ desenvolvidos no Brasil que não encontraram relação entre sexo e cuidado domiciliar, nosso estudo apontou que homens tiveram uma maior probabilidade de serem cuidados em seus domicílios. De fato, essa pode ser uma característica marcante da localidade investigada, muito embora tal resultado esteja em concordância com outro estudo brasileiro $^{17}$. Sabe-se que os homens são culturalmente cuidados por suas esposas, em muitos casos também idosas ${ }^{22}$, e que as características socioeconômicas das populações podem influenciar em tal resultado, merecendo, no entanto, novas investigações com populações semelhantes desse ponto de vista para maiores inferências conclusivas. Além disso, a condição de não ter companheiro apresentou associação positiva com cuidado domiciliar. Sabe-se que a falta de rede de apoio predispõe a autoavaliação de saúde ruim por parte do ido$\mathrm{so}^{23}$. Uma rede de apoio social inadequada é também um importante indicador de morbidade e de demais problemas de saúde, como incapacidade funcional ${ }^{2}$, que certamente aumentam a necessidade de cuidado ao idoso.

A presença de cuidador também esteve associada com a menor escolaridade. Em recentes trabalhos ${ }^{16,17}$, encontrou-se resultado semelhante, e, de fato, níveis de escolaridade mais baixos estão associados a indicadores negativos de saúde do idoso, como a incapacidade funcional ${ }^{24}$. Em contrapartida, observou-se que idosos que tinham maior nível econômico possuíam maior cuidado domiciliar. Este resultado reforça o pensamento de que, embora sejam indicadores socioeconômicos importantes, não necessariamente altos níveis econômicos e de escolaridade apontem para um mesmo desfecho. Neste caso, a associação entre maior nível econômico e cuidado domiciliar pode ser explicada pelo fato de que indivíduos com melhores condições financeiras têm a possibilidade de contratar serviço especializado a partir de um cuidador formal. Esta situação ainda é pouco frequente na realidade brasileira, mas no presente estudo, de todos os cuidadores formais relatados, cerca de $71 \%$ encontravam-se em idosos dos quartis mais elevados de nível econômico (quartis 3 e 4, respectivamente).

Inatividade física e incapacidade funcional foram fatores fortemente associados ao perfil do idoso dependente de cuidados no domicílio, assim como o evento de hospitalização no último 
ano. Sabe-se que a prática regular de atividade física é um importante comportamento a ser adotado na perspectiva de prevenir ou mesmo reduzir a presença de doenças crônicas. Além disso, indivíduos fisicamente ativos possuem maior autoestima, autoconfiança e independência funcional ${ }^{25}$.

Este estudo apresentou limitações, como o fato de não ter sido investigado o sexo do cuidador do idoso. Além disso, os dados representativos de uma região geográfica específica do município de Porto Alegre, caracterizada como área periférica e de baixo potencial socioeconômico, não permitem a extrapolação dos achados para a capital gaúcha como um todo. No entanto, comparações relativas ao perfil do idoso dependente de cuidado domiciliar são possíveis de serem realizadas com trabalhos futuros, uma vez que a ocorrência de cuidado domiciliar parece sofrer forte influência de características socioeconômicas das localidades investigadas, mas os fatores associados parecem manter consistência, em sua grande parte, com trabalhos já desenvolvidos.

Fica evidente a necessidade de ações em saúde pública com vistas a qualificar as ações prestadas por cuidadores, em sua imensa maioria, entes da própria família e sem grandes informações sobre o cuidado cotidiano do idoso. Incentivos à prevenção da ocorrência de incapacidade funcional em idosos também são bem-vindos, uma vez que essa característica mostrou-se o fator mais fortemente associado ao cuidado domiciliar.

\section{Colaboradores}

GF Del Duca propôs o tema do estudo, efetuou a análise dos dados e redigiu a primeira versão do manuscrito. AD Martinez atuou na revisão de literatura, escrita da primeira versão e revisão do manuscrito. GAN Bastos atuou na coordenação do trabalho de campo, na redação e na revisão do manuscrito.

\section{Agradecimentos}

À Coordenação de Aperfeiçoamento de Pessoal de Nível Superior pela bolsa de doutorado cedida à GF Del Duca. Fonte de financiamento: Pesquisa realizada no âmbito do Projeto Desenvolvimento de Técnicas de Operação e Gestão de serviços de Saúde em uma Região Intramunicipal de Porto Alegre - Distritos da Restinga e Extremo-Sul, de acordo com o Programa de Apoio ao Desenvolvimento Institucional do Sistema Único de Saúde (PROADI-SUS), firmado entre o Ministério da Saúde e a Associação Hospitalar Moinhos de Vento. 


\section{Referências}

1. Instituto Brasileiro de Geografia e Estatística (IBGE). Perfil dos idosos responsáveis pelos domicílios no Brasil: 2000. Rio de Janeiro: Instituto Brasileiro de Geografia e Estatística (IBGE); 2002.

2. Organização Mundial da Saúde (OMS). Envelhecimento ativo: uma política de saúde. Brasília: Organização Pan-Americana da Saúde (OPAS); 2005.

3. Lima-Costa MF, Barreto SM, Giatti L. Condições de saúde, capacidade funcional, uso de serviços de saúde e gastos com medicamentos da população idosa brasileira: um estudo descritivo baseado na Pesquisa Nacional por Amostra de Domicílios. Cad Saude Publica 2003; 19(3):735-743.

4. Siqueira FV, Facchini LA, Piccini RX, Tomasi E, Thumé E, Silveira DS, Vieira V, Hallal PC. Prevalência de quedas em idosos e fatores associados. Rev Saude Publica 2007; 41:749-756.

5. Del Duca GF, da Silva MC, Hallal PC. Incapacidade funcional para atividades básicas e instrumentais da vida diária em idosos. Rev Saude Publica 2009; 43(5):113-120.

6. Floriani CA, Schramm FR. Atendimento domiciliar ao idoso: problema ou solução? Cad Saude Publica 2004; 20(4):986-994.

7. Brasil. Lei no. 8.842 de 4 de janeiro de 1994. Dispõe sobre a Política Nacional do Idoso, cria o Conselho Nacional do Idoso e dá outras providências. Diário Oficial da União 1994; 5 jan.

8. Brasil. Lei $n^{\circ} .10 .741$ de $1^{\circ}$ de outubro de 2003. Dispõe sobre o Estatuto do Idoso e dá outras providências. Diário Oficial da União 2003; 3 out.

9. Nascimento LC. Cuidador de idosos: conhecimento disponível na base de dados LILACS. Rev Bras Enferm 2008; 61(4):514-517.

10. Associação Brasileira de Empresas de Pesquisa [ABEP]. Critério de Classificação Econômica Brasil São Paulo: ABEP; 2003.

11. Craig CL, Marshall AL, Sjöström M, Bauman AE, Booth ML, Ainsworth BE, Prott M, Ekelund U, Yngve A, Sallis JF, Oja P. International physical activity questionnaire: 12-country reliability and validity. Med Sci Sports Exerc 2003; 35(8):1381-1395.

12. Katz S, Ford AB, Moskowitz RW, Jackson BA, Jaffe MW. Studies of Illness in the Aged. the Index of Adl: a Standardized Measure of Biological and Psychosocial Function. JAMA 1963; 185(12):914-919.

13. Barros AJ, Hirakata VN. Alternatives for logistical regression in cross-sectional studies: an empirical comparison of models that directly estimate the prevalence ratio. BMC Med Res Methodol 2003; 3(1):12.

14. Veras R. Envelhecimento populacional contemporâneo: demandas, desafios e inovações. Rev Saude Publica 2009; 43(3):548-554.

Caldas CP. Envelhecimento com dependência: responsabilidades e demandas da família. Cad Saude
15. Publica 2003;19(3):773-781.

16. Giacomin KC, Uchôa E, Firmo JOA, Lima-Costa MF. Projeto Bambuí: um estudo de base populacional da prevalência e dos fatores associados à necessidade de cuidador entre idosos The Bambuí Health and Aging Study (BHAS). Cad Saude Publica 2005; 21(1):80-91.

17. Del Duca GF, Thumé E, Hallal PC. Prevalência e fatores associados ao cuidado domiciliar a idosos. Rev Saude Publica 2011;45(1):113-120.

18. Feliciano AB, de Moraes SA, de Freitas ICM. O perfil do idoso de baixa renda no Município de São Carlos, São Paulo, Brasil: um estudo epidemiológico. Cad Saude Publica 2004; 20(6):1575-1585.

19. Karsch UM. Idosos dependentes: famílias e cuidadores. Cad Saude Publica 2003; 19(3):861-866.

20. Silva LR, Vazquez-Garnica EK. El cuidado a los ancianos: las valoraciones en torno al cuidado no familiar. Texto Contexto Enferm 2008; 17(2):225-231.

21. Thumé E, Facchini A, Tomasi E, Vieira LAS. Assistência domiciliar a idosos: fatores associados, características do acesso e do cuidado. Rev Saude Publica 2010; 44(6):1102-1111.

22. Gonçalves LHT, Alvarez AM, Sena ELS, Santana LWS, Vicente FR. Perfil da família cuidadora de idoso doente/fragilizado do contexto sociocultural de Florianópolis, SC. Texto Contexto Enferm 2006; 15(4):570-577.

23. Lima-Costa MF, Firmo JOA, Uchôa E. A estrutura da auto-avaliação da saúde entre idosos: projeto Bambuí Rev Saude Publica 2004; 38(6):827-834.

24. Rosa TE, Benicio MH, Latorre Mdo R, Ramos LR. Fatores determinantes da capacidade funcional entre idosos. Rev Saude Publica 2003; 37(1):40-48.

25. Nelson M, Rejeski W, Blair S, Duncan P, Judge J, King A, Macera CA, Castaneda-Sceppa C. Physical activity and public health in older adults: recommendation from the American College of Sports Medicine and the American Heart Association. Med Sci Sports Exerc 2007; 39(8):1435-1445.

Artigo apresentado em 15/03/2011

Aprovado em 25/03/2011

Versão final apresentada em 15/04/2011 